\title{
Histological validation of ShMOLLI equilibrium contrast CMR for the measurement of diffuse myocardial fibrosis
}

\author{
Steven K White ${ }^{1,2^{*}}$, Stefan K Piechnik ${ }^{3}$, Stefan Neubauer ${ }^{3}$, Matthew D Robson ${ }^{3}$, James Moon ${ }^{1}$ \\ From 15th Annual SCMR Scientific Sessions \\ Orlando, FL, USA. 2-5 February 2012
}

\section{Background}

Diffuse myocardial fibrosis can be measured using pre and post contrast $\mathrm{T} 1$ relaxation time changes. Newer, faster sequences for T1 mapping promise whole heart coverage and improved clinical utility, but have not been validated against histology.

\section{Methods}

In fourteen patients with symptomatic severe aortic stenosis awaiting valve surgery, we performed equilibrium contrast CMR (EQ-CMR: [Flett AS et al. Circulation 2010;122(2):138-44]) to calculate $\mathrm{Vd}(\mathrm{m})$ using ShMOLLI (Shortened Modified Look-Locker Inversion recovery [Piechnik at al. JCMR 2010;12:69]) and standard multibreathold (FLASH) mapping, for the pre and equilibrium contrast T1 mapping. We compared the results to surgical biopsy.

$\mathrm{Vd}(\mathrm{m})$ was calculated by $\mathrm{Vd}(\mathrm{m})=(1$-hematocrit $) \mathrm{x} \Delta(1 /$ T1)myo $\div \Delta(1 / \mathrm{T} 1)$ blood.

Surgical left ventricular septal biopsies were fixed and stained with picrosirius red and then digitally photographed. Collagen volume fraction (CVF\%) was calculated by a blinded observer using in-house software (macro written in Image J) for automated analysis. Patients with LGE in the biopsy area were pre-specified as being excluded from analysis.

\section{Results}

FLASH T1 mapping was not possible in 2 out of 14 patients due to: 1) inability to breath hold \& 2) persistent ectopy. ShMOLLI assessment was possible in all subjects. No patient was excluded for LGE in the biopsy

\footnotetext{
'The Heart Hospital Imaging Centre, The Heart Hospital, London, UK Full list of author information is available at the end of the article
}

area, but 2 biopsy specimens were excluded because they were thought histologically to be superficial with extremes of fibrosis (patchy fibrosis).

There was a strong correlation between histological CVF\% and both FLASH Vd(m) $(\mathrm{r}=0.772, \mathrm{p}=0.021)$ and ShMOLLI Vd(m) $(\mathrm{r}=0.748, \mathrm{p}=0.007)$, as shown in Figures 1 and 2.

\section{Conclusions}

Rapid T1 mapping with ShMOLLI can be used to measure $\mathrm{Vd}(\mathrm{m})$ by EQ-CMR. The histological calibration here permits conversion of ShMOLLI Vd(m) to \% fibrosis, but also, potentially, whole heart fibrosis assessment,

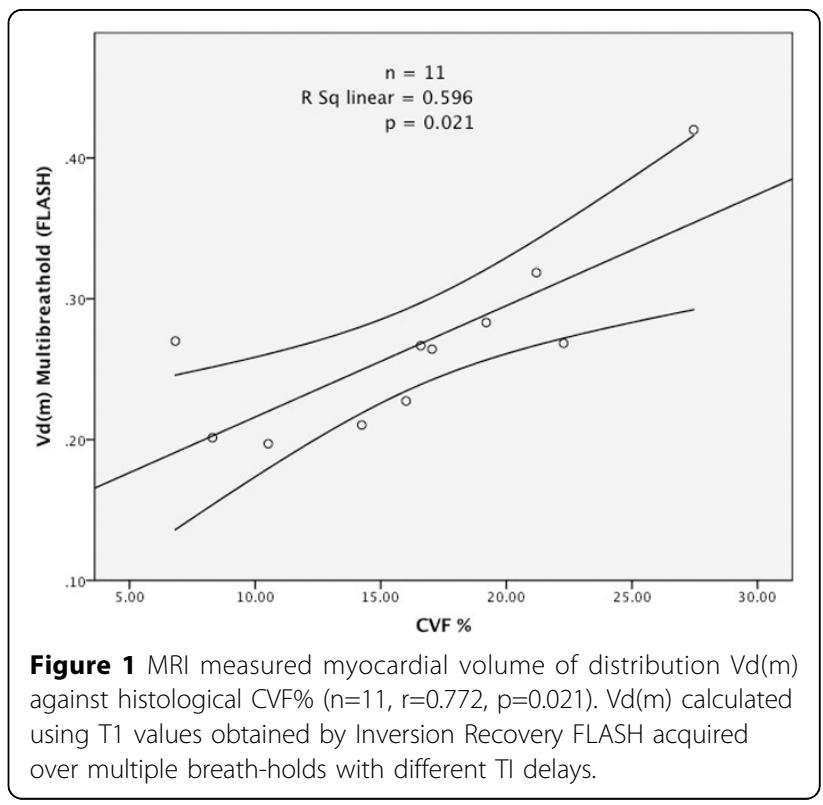




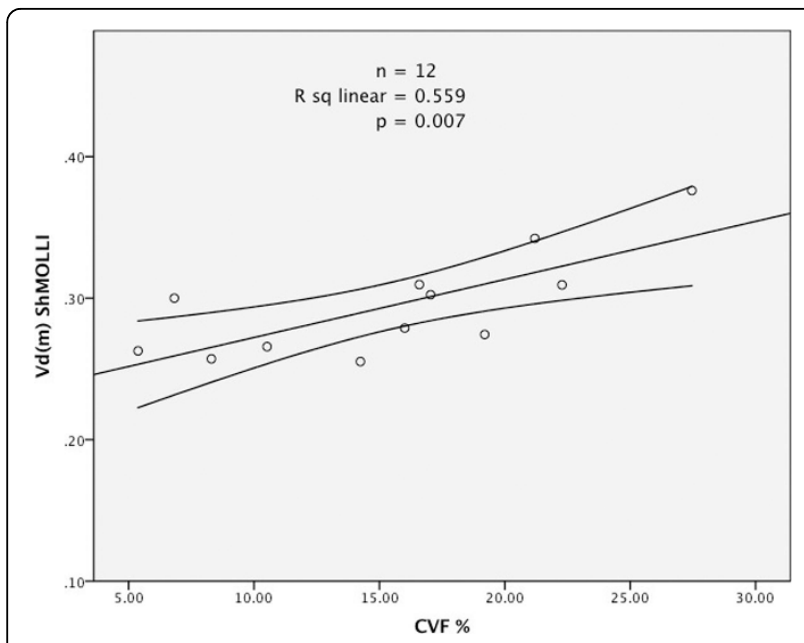

Figure 2 MRI measured myocardial volume of distribution $\mathrm{Vd}(\mathrm{m})$ against histological CVF\% ( $n=12, r=0.748, p=0.007) . V d(m)$ calculated using T1 values obtained by ShMOLLI.

and $\mathrm{Vd}(\mathrm{m})$ in patients unsuitable for slower, mutibreathold, mapping techniques.

\section{Funding}

SKW is funded by the British Heart Foundation.

SKP and MDR are funded by the NIHR Oxford Biomedical Research Centre Programme.

\section{Author details}

${ }^{1}$ The Heart Hospital Imaging Centre, The Heart Hospital, London, UK. ${ }^{2}$ The Hatter Cardiovascular Institute, University College London, London, UK.

${ }^{3}$ Oxford Centre for Clinical Magnetic Resonance Research (OCMR), University of Oxford, Oxford, UK.

Published: 1 February 2012

doi:10.1186/1532-429X-14-S1-0111

Cite this article as: White et al: Histological validation of ShMOLLI

equilibrium contrast CMR for the measurement of diffuse myocardial fibrosis. Journal of Cardiovascular Magnetic Resonance 2012 14(Suppl 1):

0111.

Submit your next manuscript to BioMed Central and take full advantage of:

- Convenient online submission

- Thorough peer review

- No space constraints or color figure charges

- Immediate publication on acceptance

- Inclusion in PubMed, CAS, Scopus and Google Scholar

- Research which is freely available for redistribution

Submit your manuscript at www.biomedcentral.com/submit 